\title{
Abundances in LMC and SMC Globular Clusters
}

\author{
Jennifer A. Johnson \\ DAO/HIA/NRC, 5071 West Saanich Road, Victoria, BC, V9E 2E\%, \\ Canada
}

\section{Inese I. Ivans}

California Institute of Technology, MS 105-24,,Pasadena, CA, 91125, $U S A$

Peter B. Stetson and James E. Hesser

DAO/HIA/NRC, 5071 West Saanich Road, Victoria, BC, V9E 2E7, Canada

\author{
Michael Bolte \\ UCO/Lick Observatory, University of California, Santa Cruz, CA \\ 95064, USA
}

\begin{abstract}
We present results for abundances in four old Magellanic Cloud clusters based on high-resolution spectroscopy of individual giants.
\end{abstract}

\section{Abundances}

We present abundances for four old LMC and SMC clusters based on highresolution spectra of 2-3 red giants per cluster taken with the Magellan telescope. We find that in the two clusters close to the LMC bar, NGC 1898 and NGC 2019, the $[\mathrm{Si} / \mathrm{Fe}]$ ratio is enhanced by $\sim 0.5$ dex relative to solar, while $[\mathrm{Ca} / \mathrm{Fe}]$ and $[\mathrm{Ti} / \mathrm{Fe}]$ are between 0 and 0.2 dex, similar to what is seen in the inner halo Milky Way clusters. In contrast, $[\mathrm{Ca} / \mathrm{Fe}]$ is $0.3 \mathrm{dex}$ in the outer LMC cluster Hodge 11 and and the SMC cluster NGC 121, which is the canonical value for the old outer halo Milky Way clusters. The $[\mathrm{Fe} / \mathrm{H}]$ values found are in good agreement with those previously derived from the slopes of the red giant branches. Finally, the $[\mathrm{Fe} / \mathrm{H}]$ and $[\alpha / \mathrm{Fe}]$ ratios are compared to the results from integrated spectra of these clusters. 